\title{
Discovery of narrow O VI emission from SN 1987A
}

\author{
George Sonneborn ${ }^{1}$, Peter Lundqvist ${ }^{2}$, Rosina C. Iping ${ }^{1,3}$, \\ and Derck L. Massa ${ }^{1,4}$ \\ ${ }^{1}$ Laboratory for Astronomy and Solar Physics, \\ NASA Goddard Space Flight Center, \\ Code 681.0, Greenbelt, MD 20771, USA \\ ${ }^{2}$ Department of Astronomy, Stockholm Observatory, \\ SE-10691 Stockholm, Sverige \\ ${ }^{3}$ Department of Physics, Catholic University of America, \\ Washington DC20064, USA \\ ${ }^{4}$ Stinger and Ghaffarian Technologies, Inc., \\ 7701 Greenbelt Road, Suite 400, Greenbelt, MD 20770, USA
}

\begin{abstract}
The 1032,1038 $\AA$ emission lines of O VI were detected from SN 1987A by the FUSE satellite observations in 2000 and 2001 . The lines are extremely narrow (FWHM $<35 \mathrm{~km} \mathrm{~s}^{-1}$ ), indicating that the emission is not a by-product of the various circums tellar shock phenomena. The most likely origin of the $\mathrm{O}$ VI emission lines is recombination of the SN progenitor's red giant wind that was photoionized by the SN shock breakout on 1987 Feb 23 .
\end{abstract}

\section{Observations and data analysis}

SN 1987A was observed by the Far Ultraviolet Spectroscopic Explorer (FUSE) satellite (Moos et al. 2000$)$ on 2000 October 7-9 $\left(41 \mathrm{ksec}\right.$, p.a. $\left.=18^{\circ}\right)$ and 2001 September $18-21\left(81 \mathrm{ksec}\right.$, p.a. $\left.=1^{\circ}\right)$. The narrow $\left(1^{\prime \prime} 25 \times 20^{\prime \prime} 0\right)$ HIRS aperture was used in order to minimize stray light from the nearby companion stars. The analysis used only the data from the $\mathrm{LiF} 1$ channel to ensure accurate aperture positioning on the SN 1987A system. The data were processed with CALFUSEv2.0.5. The O VI $1031.93 \AA$ and $1037.63 \AA$ lines are narrow (FWHM $<35 \mathrm{~km} \mathrm{~s}^{-1}$ ) and at a heliocentric radial velocity of $+280 \mathrm{~km} \mathrm{~s}^{-1}$ (Figure 1a), which places the emitting gas at rest relative to the SN (Fransson et al. 1989). These are the only spectral features in the spectral range $1000-1187 \AA$ that are attributed to SN 1987A. The fluxes for O VI 1032,1038 $\AA$ derived from the two observations are essentially the same at the two epochs $\left(F_{O \mathrm{VI}}=3.3 \pm 0.6\right.$ and $2.9 \pm 0.5 \times 10^{-15} \mathrm{erg} \mathrm{cm}^{-2} \mathrm{~s}^{-1}$ in 2000 and 2001 , respectively).

Simultaneous spectra obtained in the other FUSE apertures, positioned about $100^{\prime \prime}$ from SN 1987A, show nothing at the OVI wavelengths, eliminating diffuse emission in the LMC as a possible source of the O VI feature. The interstellar line profiles of Star 3 (1".6 from SN) show that ISM absorption (e.g., $\mathrm{O}$ VI, $\mathrm{H}_{2}$ and $\mathrm{CII}$ ) has a negligible effect on the $\mathrm{O}$ VI lines.

$\mathrm{O} V \mathrm{VI}$ is the highest ionization state yet detected in line emission from the SN 1987A circumstellar material. The narrow width of the lines indicates that 

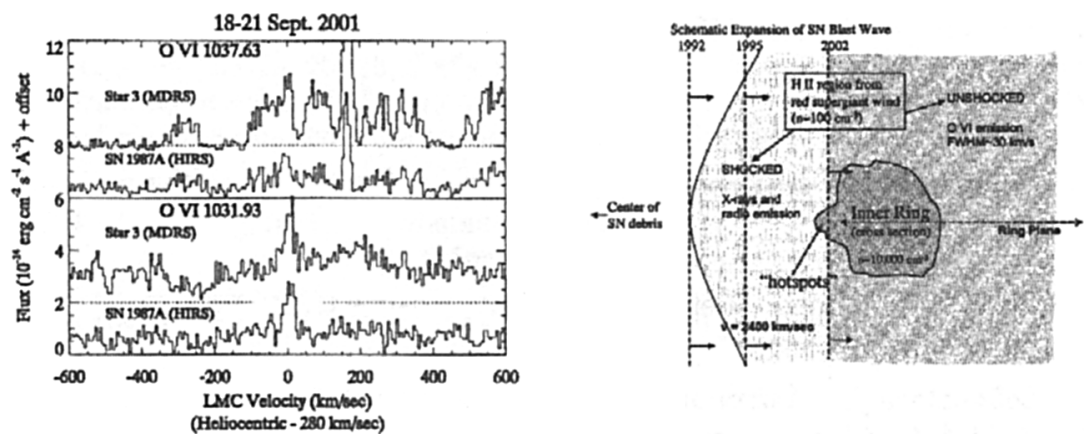

Figure 1. FUSE spectra of SN 1987A and Star 3 showing narrow O VI emission (left), and a cartoon of the CS environment (right).

the O VI emission does not originate in shocked gas where the $\mathrm{SN}$ debris collides with circumstellar material (FWHM Ly $\alpha \approx 10000 \mathrm{~km} \mathrm{~s}^{-1}$ ). This is the remnant of mass lost in the red giant phase of the progenitor (Sonneborn et al. 1998). There are also at least 16 hot spots (R. Kirshner, private communication) where the forward shock is colliding with the dense inner ring $\left(n_{\mathrm{e}}>20000 \mathrm{~cm}^{-3}\right.$, Fransson et al. 1989; Sonneborn et al. 1997). Emission from these hot spots have FWHM $=200-400 \mathrm{~km} \mathrm{~s}^{-1}$ (Michael et al. 2000).

The $\mathrm{O}$ VI emission probably originates from unshocked gas in the $\mathrm{H}$ II region of very low density $\left(n_{\mathrm{e}}<100 \mathrm{~cm}^{-3}\right)$ material near the inner circumstellar ring that was ionized by the supernova outburst in 1987. This is the unshocked part of the same material that the SN debris is colliding with to produce the Ly $\alpha$ emission (see Figure 1b). It is unlikely that the narrow OVI emission is the result of recent re-ionization of circumstellar gas by X-rays produced by shock interactions. The increasing X-ray flux would be accompanied by an increase in O VI flux, which is not indicated by the present observations. The observed O VI fluxes are in agreement with recombination models (Lundqvist 1999) for the circumstellar material, after the observed O VI fluxes are corrected for interstellar extinction. Additional FUSE observations are planned to follow the development of the O VI emission.

Acknowledgments. This work is based on data obtained by the NASACNES-CSA FUSE mission for the FUSE Guaranteed Time Team, supported in part by contract NAS5-32985 to The Johns Hopkins University. FUSE is operated for NASA by JHU under the same contract. RCI acknowledges support for grant NAG5-11137 to the Catholic University of America.

\section{References}

Fransson, C., Cassatella, A., Gilmozzi, R., et al. 1989, ApJ 336, 429

Lundqvist, P., 1999, ApJ 511, 389

Michael, E., McCray, R., Pun, C.S.J., et al. 2000, ApJ (Letters) 542, L53

Moos, H.W., Cash, W.C., Cowie, L.L., et al. 2000, ApJ (Letters) 538, L1

Sonneborn, G., Fransson, C., Lundqvist, P., Cassatella, A., Gilmozzi, R., Kirshner, R.P., Panagia, N., Wamsteker, W. 1997, ApJ 477, 848 